\title{
Opinion
}

\section{The Labile Limits of Forbidden Interactions}

Juan P. González-Varo ${ }^{1,2, *}$ and Anna Traveset ${ }^{3}$

4

${ }^{1}$ Conservation Science Group, Department of Zoology, University of Cambridge, Cambridge, UK

$6{ }^{2}$ Integrative Ecology Group, Department of Integrative Ecology, Estación Biológica de Doñana (EBD-CSIC), Sevilla, Spain

$8{ }^{3}$ Mediterranean Institute of Advanced Studies, IMEDEA (CSIC-IUB), Esporles, Mallorca, Spain

*Correspondence: jpg62@cam.ac.uk (J.P. González-Varo).

Keywords: biotic interactions; ecological networks; intraspecific variability; trait matching 
Forbidden links are defined as pairwise interactions that are prevented by the biological traits of the species. Here, we focus on the neglected importance of intraspecific trait variation in the forbidden link concept. We show how intraspecific trait variability at different spatiotemporal scales, and through ontogeny, reduces the expected prevalence of forbidden interactions. We also highlight how behaviour can foster interactions that, from traits, would be predicted as forbidden. We therefore discuss the drawbacks of frameworks recently developed to infer biotic interactions using available trait data (mean values). Mispredictions can have disproportionate effects on inferences about community dynamics. Thus, we suggest including intraspecific trait variability in trait-based models and using them to guide the sampling of real interactions in the field for validation.

\section{What are forbidden interactions and why do they matter?}

Biotic interactions across trophic levels govern the dynamics of communities and the functioning of ecosystems [1-5]. Interspecies relationships not only determine energy fluxes [6], but also mediate key ecological functions such as mycorrhizal-mediated mineral nutrition [7], animal-mediated pollination and seed dispersal [8]. When sampling such interactions in the field, the norm is that certain pairwise interactions remain undetected, named the missing and forbidden links [9,10]. Missing links (see Glossary) are possible but hardly observable interactions, whereas forbidden links are pairwise interactions that are prevented by biological traits of the species $[9,10]$, such as their size, morphology, phenology, physiology-biochemistry or habitat specificity. For instance, large-sized food items, like animal preys or fruits, can preclude consumption by small-gaped eaters [11-15], whereas non-overlapping phenologies impede the temporal encounter between potentially

interacting species [10]. Indeed, both morphology and phenology explain a huge fraction of unobserved interactions in pollination and frugivory networks [10]. By constraining the number of possible interactions, forbidden interactions are of major importance for the 
structure and functioning of ecological networks, including food webs and bipartite networks of mutualistic and antagonistic systems [16-22].

Largely motivated by the ongoing biodiversity crisis, ecologists have recently stressed the need for inferring biotic interactions at wide geographical scales [15,23-26], acknowledging interactions are key for predicting species distributions in response to global change pressures [27-29]. To cope with this demand, Morales-Castilla et al. [24] have recently presented a new conceptual framework to infer pairwise interactions in unknown systems, that is, the elements that build up complex networks of ecological relationships. The framework is based on trait matching, particularly, on the use of available trait data (e.g. body size) to establish forbidden links [24]. A critical question that arises from such predictive attempts is: how accurately can we establish forbidden links using available trait information? In other words, how reliably can we infer different types of pairwise interactions using available trait data?

Here, we focus on the neglected importance of intraspecific trait variability in the forbidden link concept, which is usually considered as a categorical fact (forbidden or not) rather than as a trait-matching continuum. Matching traits can vary dramatically within a species across space (see Box 1), between individuals within populations and between populations within its whole distribution range [30-33]. But matching traits can vary even more in time (Box 1), between years and through the ontogenetic stages of individuals. We show how intraspecific trait variability at different spatiotemporal scales reduces the expected prevalence of forbidden interactions (Box 1), thereby increasing species degree and overall network connectance. We also highlight that behaviour can foster pairwise interactions that would rarely be expected from trait data. Consequently, we discuss the potential limits and drawbacks of using mean values available from trait databases [34-36] to infer pairwise interactions and community dynamics. Although the trait-matching 
approach can be useful for elucidating general patterns within already sampled systems, we argue that it could fail to detect key interactions that allow species co-occurrences and drive critical ecological functions.

\section{How accurately can we assign forbidden links using available trait data?}

The forbidden link concept has proved useful to explain a posteriori interaction degree and some emergent properties of empirical networks [10,21,37]. In these analyses, forbidden links are regarded as immutable realities within the studied systems, i.e. as structural zeros in the adjacency matrices of interacting species $[10,21,24]$. This rigid view has probably been fostered by strong trait-matching evidence from networks in which interaction sampling was coupled with trait measurements [10,21,37]. However, it is widely recognized that empirical networks represent temporal snapshots (weeks, months, or at best a few years) of complex interactions sampled at a particular site [38]. Because traits are a suitable post hoc explanation of interaction patterns in these 'well-known' snapshots [37], can we then use traits to accurately infer pairwise interactions in unknown systems?

The framework proposed by Morales-Castilla et al. [24] relies on the use of available trait information (or phylogenetic distance as a surrogate for trait similarity if information on traits is unavailable). Global databases of functional traits are currently accessible for different taxonomic groups, including plants, birds, mammals, reptiles and amphibians [3436]. These data consist of trait mean values, which should ideally represent averages for all individuals of each species across its whole distribution range. The reality is that trait means usually account for a small number of individuals measured at a particular location in a certain moment. But more limiting than their representativeness is the fact that trait means neglect the existing intraspecific variation, thus, they underestimate the degree of trait matching by overestimating the incidence of forbidden interactions (Figure 1, Box 1). 
The latter can lead to serious misinterpretations about the dynamics and functioning of the inferred ecosystems.

$$
\text { Conspecific individuals differ in the multiple traits that produce their phenotypes. }
$$

Such variability was indeed the essence for the Darwin's theory of evolution by natural selection and is considered to be crucial in many ecological and evolutionary processes [3941]. Intraspecific trait variability enhances average interaction degree of species and network connectance (Figure 1, Box 1), thereby robustness to secondary extinctions $[42,43]$. In the following sections, we address the role of different sources of intraspecific variation on the degree of trait matching between species. Moreover, we discuss the importance of behaviour in allowing interactions that would be predicted as forbidden on the basis of available traits.

\section{Matching traits vary in space and time}

The most pervasive cases of intraspecific trait variation are among-individual differences in age and sex $[39,44]$. We specifically focus on ontogenetic trait variation in the next section, since traits and interactions can vary tremendously between individuals of different age or life stage. Sexual dimorphisms typically entail differences in size or shape, which can lead each gender to interact with different species $[45,46]$. For instance, males and females of the purple-throated carib hummingbird (Eulampis jugularis) feed on two different Heliconia species whose flowers match to the shape and length of the bill of each gender [45]. In the Eurasian sparrowhawk (Accipiter nisus), the body size of females (185-342 g) is about double that of males (110-196 g) [47]. As a result, females prey on birds up to 150 g (occasionally up to $500 \mathrm{~g}$ ), mainly thrushes (Turdidae), starlings (Sturnidae) and pigeons (Columbidae), whereas males normally prey on birds up to $40 \mathrm{~g}$, including tits (Paridae), finches (Fringillidae), sparrows (Passeridae) and buntings (Emberizidae) [48]. Both examples illustrate how sexual dimorphism increases intraspecific trait variability, thus, 
interaction degree at the species level. Hence, some interactions can be forbidden for one gender but possible for the other. Yet, sex-dependent trophic niches with bimodal trait distribution are reduced to a single value in trait databases: the mean.

Furthermore, intraspecific trait variability can also be huge among individuals of the same sex and age. Species traits vary in space at different hierarchical levels or spatial scales $[30,32,33]$, among individuals within populations and among populations across the species ranges (see Figure I in Box 1). In the case of plants, traits even vary within the same individual (see Figure II in Box 1), among leaves, flowers and fruits, organs that mediate several interactions with different types of feeding animals (herbivores, pollinators, florivores, seed dispersers and seed predators) [31,49]. Phenotypes result from the interaction between individuals' genes and local environmental conditions, each source of variation having a specific relative weight in determining the expression of different traits [50]. Therefore, those traits that strongly depend on the local environment are prone to vary in time, between phenological events under different climate conditions (Box 1). For instance, water deficits reduce the size and alter the chemical composition of fruits [51]. Consequently, these traits can hugely vary between consecutive years even in the same individual plant, thus, in the same population, which increases uncertainty even when using local trait data to infer local interactions (Box 1). Despite its importance, intraspecific trait variability in time has been largely overlooked in community ecology [25,41].

Regardless of whether the main source of variation is genetic or environmental, trait variability makes individuals of a given species (or plant organs) non-interchangeable from the perspective of their interacting partners (see Figure II in Box 1). Actually, different bird species have been reported to consume fruits of specific size distributions within the same plant species according to their gape widths $[13,52]$. Similarly, different flower visitors and herbivores have been documented to visit different individual plants according to their 
flowering phenology, morphology and flower scent [53]. At the species level, the result of particular individuals (or plant organs) interacting with particular partner species is an increase in the possibilities of trait matching (see Figure II in Box 1), that is, a broader 'interaction niche' generated by between-individual niche differences [39]. Whenever inheritable, such differences can also be the basis of evolutionary changes after the loss or gain of partner species [13].

Conversely, a framework that uses trait means to infer biotic interactions assumes that all individuals, thereby all populations, are interchangeable. This assumption can lead to important predictive errors associated to $(i)$ the representativeness of available trait data and (ii) the neglect of intraspecific variation. First, trait means account for individuals sampled in one or a few locations during a particular moment. How well do these subsets represent a species average across its entire distribution? For example, mean diameter in myrtle (Myrtus communis) fruits can range from 7.3 to $10.4 \mathrm{~mm}$, depending on which population and year the fruits were sampled (see Figure I in Box 1). Hence, using a trait mean obtained at a particular 'site-year' combination to infer interactions at different locations can lead to under- or overestimation of forbidden links, depending on the sign of the extrapolation error (e.g. from population L to $M$ or vice versa; Figure I in Box 1). Secondly, using trait means raises concerns about the predicted prevalence of forbidden interactions when neglecting intraspecific variability. In the same example, myrtle fruits are predicted as fully prevented for Sardinian warblers (Sylvia melanocephala) when using mean diameters as interaction threshold, but such interaction is expected across myrtle populations when using minimum values as threshold (see Figure I in Box 1). Accordingly, there is empirical evidence that, indeed, Sardinian warblers regularly consume myrtle fruits in different regions $[10,54,55]$. The myrtle and the Sardinian warbler are common and locally abundant species in the studied sites, and abundance is a critical factor promoting 
both the establishment and strength of interactions through increasing interspecies encounter rates $[25,56]$, even when such interactions are predicted to be unlikely from traits.

\section{Ontogeny enlarges intraspecific trait variability}

Traits can hugely vary between the life stages of one species, which promotes stagespecific interactions $[44,57]$. The first life stage of most organisms (e.g. seeds in plants, and eggs or newborn in animals) is normally tiny compared to adults. Thenceforth, ontogeny normally entails an increase in body size, thereby an allometric shift of interacting partners [12,57-59]. Keeping species roles as predators and prey, ontogeny not only allows young prey individuals to be consumed by smaller predator species (e.g. Figure 2a), but also adult and young predator individuals to feed on different prey species according to their size $[12,14,58,59]$. Therefore, ontogeny generates a broader niche space for both consumers and resources, allowing interactions that would be assigned as forbidden if we only look at the adult traits. Besides, individuals of some species can increase in weight by several orders of magnitude during their life, shifting their role from being a prey of certain species to being a predator of another species (Figure $2 b$ ). These vast shifts can promote reciprocal predation among species of the same trophic level and even cannibalism $[14,57,59,60]$. For instance, an egg of Nile crocodile (Crocodylus niloticus) weights $\sim 0.1 \mathrm{~kg}$ while an adult typically exceeds $200 \mathrm{~kg}$, i.e. four orders of magnitude larger [61,62]. Ontogenetic variation allows animals of ca. 1-10 kg such as monitor lizards (Varanidae), herons (Ardeidae) and ravens (Corvidae) to prey on the eggs and juveniles of this apex predator (Figure 2a), with profound effects on its demography $[61,63]$. Interestingly, ontogeny can reverse the direction of predation when adult predators can feed on those species which predate on their early life stages, leading to feeding loops (Figure 3). Such loops are remarkably common and can be of major importance for community dynamics $[14,57,59,60]$. 
It is not difficult to envisage that ontogenetic variability can generate an enormous uncertainty when attempting to predict biotic interactions using available trait means of

194

adult individuals, which might lead us to wrong inferences about community dynamics. For instance, reciprocal predation through life-stage-specific interactions promotes coexistence between native Mohave tui chub (Siphateles bicolor mohavensis) and invasive western mosquitofish (Gambusia affinis) [14]. Gravel et al. [26] have recently proposed distinguishing 'subspecies' by size (ontogenetic) categories to build trait-based networks, which would describe more accurately those pairwise interactions involving species with size structured populations. A major limitation to this approach is the lack of available trait data for species' life-stages other than the adult [34-36].

\section{Behaviour can allow 'forbidden' interactions}

Species interactions that initially appear unlikely can eventually be established at ecological timescales through changes in animal behaviour. Animal innovations (i.e. animals behaving in an innovative way) have been described in a number of taxa, particularly in resourcepoor or novel environments [64]. For example, Laland \& Reader [65] found that fooddeprived guppies (Poecilia reticulata) are more likely to develop foraging innovations than non-food-deprived individuals. Tinker et al. [66] found analogous results in California sea otters (Enhydra lutris nereis) at a resource-limited site, where individuals specialized on different preys by acquiring specific behavioural skills. Birds are especially innovative in their foraging techniques [67] and recent experiments have demonstrated that the information can spread rapidly among individuals in the population through social network ties [68]. The kelp gulls (Larus dominicanus) at Peninsula Valdés provide an excellent example. In the 1970s, kelp gulls began to feed on skin and blubber of living southern right whales (Eubalaena australis) by pecking on their backs [69]. Since then, the percentage of living right whales (mothers and calves) with gull lesions has increased from $2 \%$ to $99 \%$ in 
the 2000s, and wounding is now considered a major cause of whale mortality [70]. The gull population grew rapidly in the last decades parallel to the human population on the nearby mainland [69]. Rapid population growth is a common feature of biological invasions, and this example shows that the outcomes can be surprising in terms of interspecies interactions. Even more unexpected is the emergence of interactions between the sharpbeaked finch (Geospiza difficilis) and different booby species (Sula spp.) on the small, isolated and food-limited islands of Wolf and Darwin (see Figure 2c). This finch feeds on insects and small seeds in the Galapagos Islands, but on Wolf and Darwin the so-called vampire finch also feeds on the blood and eggs of these seabirds [71]. Information on bill traits alone would rarely predict this strange but critical interaction for the sharp-beaked 228 finch at the extreme of the archipelago.

Besides promoting interactions, behaviour can also modify the sign of pairwise interactions, from mutualistic (positive) to antagonistic (negative) or vice versa, leading to partner dualities. For instance, scatter-hoarding birds and mammals are seed predators but they also provide effective seed dispersal by moving caches that are eventually forgotten or released if the animal dies [72]. In the Neotropics, agoutis (Dasyprocta punctata) are actually claimed to have been responsible for the persistence of large-seeded species that were once dispersed by megafauna which went extinct more than 10,000 years ago [73]

(Figure 2d). It is not always simple to foresee in which cases an animal species will behave as a mutualist or antagonist, but we must bear in mind that such changes in the sign of 238 interactions can have important implications for community dynamics.

\section{Concluding Remarks and Future Directions}

The thresholds delimiting forbidden interactions are labile as a result of intraspecific trait variability, which can be substantial. We have illustrated that only using available trait data 
(i.e. means) underestimates species degree (thus, network connectance) through overestimating the incidence of forbidden links. Also, that available trait means might not be representative across the species ranges, which can lead to both over- and underestimations of interactions inferred locally. Recently developed probabilistic models now allow incorporating intraspecific trait variability to predict interactions $[26,56]$. However, the challenge to accurately infer biotic interactions in unknown or novel ecosystems is to gather detailed trait data for multiple species, well replicated across their ranges and ontogenetic stages, and then, to make these data available alongside descriptive statistics of intraspecific trait variability. Although promising, this enterprise is clearly colossal. Alternatively, one could quantify intraspecific trait variability in well-sampled species (e.g. Box 1) and transfer an equivalent amount of variance to taxonomically or phylogenetically close species lacking such detailed data (see Outstanding Questions). In sum, we recommend incorporating intraspecific trait variability in models predicting networks of biotic interactions.

Even with detailed trait information available, there are still many sources of uncertainty affecting the establishment of pairwise interactions. On the one hand, predicted but not recorded interactions (false positives) could reflect mismatches in nonmorphological or phenological traits (such as physiology or biochemistry), or reflect cognitive constraints, which can block the formation of inferred interactions [e.g. 74]. On the other hand, in novel scenarios, behavioural innovation can promote interactions predicted as forbidden (false negatives). Evidence shows that these 'outliers' matter, especially when they affect common or keystone species with a disproportionate role in community dynamics and ecosystem functioning (see Outstanding Questions). biotic interactions to trait-matching models can critically limit our inferences. Yet, inferred 
networks might serve as 'maps' to guide sampling of real interactions. Particularly, they could help to focus sampling effort on predicted links (included the forbidden ones) that seem to be key for the target communities, in order to validate network-model predictions (see Outstanding Questions). Novel techniques (e.g. DNA barcoding, GPS tagging, camera trapping) are now revolutionizing the sampling of biotic interactions, unravelling unprecedented information about the functions they entail $[52,73,75]$. We thus need synergies between trait-based models and empirical studies if we are to understand how interspecies relationships shape the fate of communities and ecosystems.

\section{Acknowledgments}

We thank José M. Costa, Pedro Jordano, Rúben Heleno and Carlos M. Herrera for kindly providing bird gape width data. Francisco Rodríguez-Sánchez and Néstor Pérez-Méndez provided feedback on the first ideas of this paper. Lynn Dicks and Benno Simmons reviewed the English and, together with Beatriz Rumeu, provided useful comments on manuscript drafts. Three anonymous reviewers contributed with constructive suggestions that greatly improved the paper. While writing this paper, J.P.G.V. was funded by a postdoctoral fellowship from the Severo-Ochoa Program for Centres of Excellence in R\&D\&I (SEV-2012-0262) and an Individual Fellowship from the Marie Sklodowska-Curie Actions (H2020-MSCA-IF-2014), whereas A.T was funded by project CGL201318759BOS from the Spanish Government. 
Figure 1. Frequency Distribution of Matching Traits (e.g. Body Size or Phenology) in a Consumer and a Resource Species. Interaction is possible whenever $x_{\text {consumer }} \geq x_{\text {resource. }}$ (a) Mismatching between both trait means and intraspecific variability prevent interaction, leading to a totally forbidden link. (b) Mismatching occurs between trait means but intraspecific variability allows interaction, leading to a partially forbidden link. The difference between (a) and (b) - thus, our ability to infer interactions - may depend on how broadly intraspecific trait variability has been assessed in space and time (see Box 1).

Figure 2. Examples of How Ontogenetic Variability and Behaviour Promote Interspecific Interactions or Change their Sign. (a) Small groups of golden jackals (Canis aureus), which are two orders of magnitude smaller than an adult blue wildebeest (Connochaetes taurinus), can hunt wildebeest calves [62]. (b) The Nile monitor (Varanus niloticus), three orders of magnitude smaller than an adult Nile crocodile (Crocodylus niloticus), is the main predator of crocodile eggs [61]. (c) The Galápagos sharp-beaked finches (Geospiza difficilis) mainly feed on insects and small seeds, but on the small and food-limited islands of Wolf and Darwin, they cut wounds on seabirds (boobies Sula spp.) to drink their blood; they also push and roll seabird eggs into rocks to break them and feed on them [71]. (d) The Central American agouti (Dasyprocta punctata), a seed predator (antagonist) of the black palm (Astrocaryum standleyanum), can effectively disperse its seeds through scatter-hoarding, acting as a mutualistic partner [73]. Photos reproduced with permission from Todd Gustafson (a), Anup Shah (b), Pete Oxford (c) and Christian Ziegler (d).

Figure 3. Feeding Loop Generated by Ontogenetic Reversal of Predation. Arrows, from prey to predator, denote predation. (a) Species level diagram showing reciprocal predation between species $j$ and $k$. (b) Life-stage level diagram showing how reciprocal predation is mediated by stagespecific interactions between both predators during their ontogeny: species $j$ is a prey of species $k$ during its earlier life stages, but it becomes a predator of species $k$ in the adult stage. We could hardly infer the role of species $k$ as predator of species $j$ using trait data of adult individuals (e.g. body size). 


\section{Box 1. Spatiotemporal variation in trait matching}

Assessing spatiotemporal variation in size matching between the fruits of the Mediterranean myrtle (Myrtus communis) and the gape of the five most abundant frugivore species in CircumMediterranean woodlands during fruit ripening (see details in Appendix S1). We assess variation in fruit diameter among populations and years across the distribution range of the plant (Figure I), and among individual plants and between consecutive years within three of these populations (Figure II) (see sections A and B, respectively, in Appendix S1). A gape width smaller than a fruit diameter involves the incapability of the frugivore to swallow the fruit $[11,13]$, and thus a forbidden interaction. However, different forbiddance thresholds in fruit diameter - mean, first quartile $\left(Q_{25}\right), 5^{\text {th }}$ percentile $\left(\mathrm{P}_{05}\right)$ or minimum - can lead to different prevalences of forbidden interactions. These thresholds represent the capability of an average individual of each frugivore species (mean gape width) to consume fruits of average size (mean), or to consume at least $25 \%\left(Q_{25}\right), 5 \%\left(P_{05}\right)$, or some (minimum) of available fruits. We use this mixed and conservative approach, using mean values for the frugivores but intraspecific variability for the plant [41], owing to the lack equivalent geographical variation for gape width data [10,76-78] (see section C in Appendix S1). We show how fruit diameter varies geographically and temporally, among populations and between years within populations (Figure I). Due to geographical variation (15\% variance), some populations are predicted as forbidden for narrow-gaped frugivores while others do not (Figure I). But due to interannual variation (22\% variance), some populations are predicted as forbidden some years but not others (e.g. populations $D$ and $M$ in Figure I). We also show how forbidden links largely drop when using less restrictive thresholds. For example, when using mean fruit diameter as threshold, S. melanocephala results a forbidden frugivore in $100 \%$ of population-year combinations, but only in $26 \%$ when using the minimum diameters (Figure I). Accordingly, empirical information from different frugivory studies shows that all five species - even those with the smallest gape widths - actually consume myrtle fruits in different sites $[10,54,55]$.

\section{Figure I}


Similarly, we show how fruit diameter varies among individuals within populations and between consecutive years (Figure II). Due to among-individual variation (56\% variance), some plants are predicted as forbidden for narrow-gaped frugivores while others do not. But due to interannual variation ( $31 \%$ variance), some plants are predicted as forbidden some years while not in others (Figure II). Again, the prevalence of forbidden links depends on the forbiddance thresholds chosen (Figure II).

\section{Figure II}

Figure I. Geographical and Temporal Variation in Trait Matching. (a) Boxplot (median, quartiles and $5^{\text {th }} / 95^{\text {th }}$ percentiles) showing variation in fruit diameter across 18 populations of Mediterranean shrub Myrtus communis, some of them sampled in different years (27 population-year combinations) (Appendix S1). Black dots denote means. White dots denote minimum values. Horizontal lines denote the mean gape width $(\mathrm{mm})$ of the five most abundant frugivore species in Circum-Mediterranean woodlands during the winter (Er: Erithacus rubecula; Sa: Sylvia atricapilla; Sm: Sylvia melanocephala; Tm: Turdus merula; Tp: Turdus philomelos), period when M. communis ripe its fruits. (b) Map of the Mediterranean Basin with the location of the study M. communis populations. The distribution of $M$. communis is shown in dark-grey (redrawn from Migliore et al. [79]).

Figure II. Intra- and Interindividual Variation in Trait Matching. Boxplot showing variation in fruit diameter among marked myrtle plants in two consecutive years (grey and white boxes, respectively) and in three different populations. Black dots denote means. Grey and white dots denote minimum values. Horizontal lines denote the mean gape width $(\mathrm{mm})$ of the five most abundant frugivore species in Mediterranean woodlands during the winter (bird species acronyms as in Figure I). 


\section{Glossary}

Bipartite network: ecological network comprising two trophic levels (e.g. host and parasitoids, plants and pollinators, or plants and frugivores) with the links between species representing trophic interactions (i.e. fluxes of matter and energy) and ecological functions (e.g. pollination, seed dispersal) $[8,9]$.

Connectance: the fraction of pairs of species that interact in an ecological network $[42,80]$. In food webs, $C=L / S^{2}$, where $L$ is the number of interactions (links) and $S$ the number of species in the community. In bipartite networks, $C=L / S_{r} S_{c}$, where $S_{r}$ and $S_{c}$ are the number of species in each trophic level (resources and consumers; e.g. plants and pollinators).

Food web: ecological network comprising multiple trophic levels (e.g. primary producers, herbivores, predators, detritivores) with the links between species representing trophic interactions (i.e. fluxes of matter and energy) [17].

Forbidden link: pairwise interaction that is prevented by the biological traits of the species $[9,10]$.

Functional trait: any trait affecting, directly or indirectly, the performance and fitness of individuals [41].

Missing link: possible but hardly observable pairwise interaction (e.g. between rare species), thus, a result of under-sampling [10].

Species degree: the number of different species a certain species interacts with in an ecological network $[9,42]$.

Trait: any morphological, physiological, phenological or behavioural feature measurable at the individual level [41].

Trait matching: phenotypic expression of functional traits that allow a particular interspecific interaction (Figure 1). 


\section{References}

1. Roemer, G.W. et al. (2002) Golden eagles, feral pigs, and insular carnivores: How exotic species turn native predators into prey. Proc. Natl. Acad. Sci. USA 99, 791796.

2. Cottenie, K. and De Meester, L. (2004) Metacommunity structure: Synergy of biotic interactions as selective agents and dispersal as fuel. Ecology 85, 114-119.

3. Ripple, W.J. and Beschta, R.L. (2012) Trophic cascades in Yellowstone: The first 15years after wolf reintroduction. Biol. Conserv. 145, 205-213.

4. García, D. and Martínez, D. (2012) Species richness matters for the quality of ecosystem services: a test using seed dispersal by frugivorous birds. Proc. R. Soc. Lond., Ser. B: Biol. Sci., doi:10.1098/rspb.2012.0175.

5. Thompson, R.M. et al. (2012) Food webs: reconciling the structure and function of biodiversity. Trends Ecol. Evol. 27, 689-697.

6. Brose, U. et al. (2008) Foraging theory predicts predator-prey energy fluxes. J. Anim. Ecol. 77, 1072-1078.

7. Selosse, M.-A. et al. (2006) Mycorrhizal networks: des liaisons dangereuses? Trends Ecol. Evol. 21, 621-628.

8. Bascompte, J. and Jordano, P. (2007) Plant-animal mutualistic networks: The architecture of biodiversity. Annu. Rev. Ecol., Evol. Syst. 38, 567-593.

9. Jordano, P. et al. (2003) Invariant properties in coevolutionary networks of plantanimal interactions. Ecol. Lett. 6, 69-81.

10. Olesen, J.M. et al. (2011) Missing and forbidden links in mutualistic networks. Proc. R. Soc. Lond., Ser. B: Biol. Sci. 278, 725-732.

11. Wheelwright, N.T. (1985) Fruit-size, gape width, and the diets of fruit-eating birds. Ecology 66, 808-818.

12. Brose, U. et al. (2006) Consumer-resource body-size relationships in natural food webs. Ecology 87, 2411-2417.

13. Galetti, M. et al. (2013) Functional extinction of birds drives rapid evolutionary changes in seed size. Science 340, 1086-1090.

14. Henkanaththegedara, S.M. and Stockwell, C.A. (2014) Intraguild predation may facilitate coexistence of native and non - native fish. J. Appl. Ecol. 51, 1057-1065.

15. Alofs, K.M. and Jackson, D.A. (2015) The vulnerability of species to range expansions by predators can be predicted using historical species associations and body size. Proc. R. Soc. Lond., Ser. B: Biol. Sci. 282.

16. Cohen, J.E. et al. (2005) Body sizes of hosts and parasitoids in individual feeding relationships. Proceedings of the National Academy of Sciences of the United States of America 102, 684-689. 
17. Woodward, G. et al. (2005) Body size in ecological networks. Trends Ecol. Evol. 20, 402-409.

18. Bluthgen, N. et al. (2006) Measuring specialization in species interaction networks. BMC Ecol. 6, 9.

19. Santamaría, L. and Rodríguez-Gironés, M.A. (2007) Linkage rules for plantpollinator networks: Trait complementarity or exploitation barriers? PLOS Biol. 5, e31.

20. Allesina, S. et al. (2008) A general model for food web structure. Science 320, 658661.

21. Vizentin-Bugoni, J. et al. (2014) Processes entangling interactions in communities: forbidden links are more important than abundance in a hummingbird-plant network. Proc. R. Soc. Lond., Ser. B: Biol. Sci. 281.

22. Lever, J.J. et al. (2014) The sudden collapse of pollinator communities. Ecol. Lett. 17, 350-359.

23. Kissling, W.D. and Schleuning, M. (2015) Multispecies interactions across trophic levels at macroscales: retrospective and future directions. Ecography 38, 346-357.

24. Morales-Castilla, I. et al. (2015) Inferring biotic interactions from proxies. Trends Ecol. Evol. 30, 347-356.

25. Poisot, T. et al. (2015) Beyond species: why ecological interaction networks vary through space and time. Oikos 124, 243-251.

26. Gravel, D. et al. (2013) Inferring food web structure from predator-prey body size relationships. Methods Ecol. Evol. 4, 1083-1090.

27. Araújo, M.B. and Luoto, M. (2007) The importance of biotic interactions for modelling species distributions under climate change. Global Ecol. Biogeogr. 16, 743-753.

28. Heikkinen, R.K. et al. (2007) Biotic interactions improve prediction of boreal bird distributions at macro-scales. Global Ecol. Biogeogr. 16, 754-763.

29. Schweiger, O. et al. (2008) Climate change can cause spatial mismatch of trophically interacting species. Ecology 89, 3472-3479.

30. Blanck, A. and Lamouroux, N. (2007) Large-scale intraspecific variation in lifehistory traits of European freshwater fish. J. Biogeogr. 34, 862-875.

31. Herrera, C.M. (2009) Multiplicity in unity: plant subindividual variation and interactions with animals. University of Chicago Press.

32. Albert, C.H. et al. (2010) A multi-trait approach reveals the structure and the relative importance of intra- vs. interspecific variability in plant traits. Funct. Ecol. 24, 1192-1201.

33. Messier, J. et al. (2010) How do traits vary across ecological scales? A case for trait - based ecology. Ecol. Lett. 13, 838-848. 
34. Jones, K.E. et al. (2009) PanTHERIA: a species-level database of life history, ecology, and geography of extant and recently extinct mammals. Ecology 90, 26482648.

35. Kattge, J. et al. (2011) TRY - a global database of plant traits. Global Change Biol. $17,2905-2935$.

36. Wilman, H. et al. (2014) EltonTraits 1.0: Species - level foraging attributes of the world's birds and mammals. Ecology 95, 2027-2027.

37. Eklöf, A. et al. (2013) The dimensionality of ecological networks. Ecol. Lett. 16, 577-583.

38. Olesen, J.M. et al. (2010) From Broadstone to Zackenberg: Space, time and hierarchies in ecological networks. Adv. Ecol. Res. 42, 1-69.

39. Bolnick, D.I. et al. (2003) The ecology of individuals: incidence and implications of individual specialization. The American Naturalist 161, 1-28.

40. Bolnick, D.I. et al. (2011) Why intraspecific trait variation matters in community ecology. Trends Ecol. Evol. 26, 183-192.

41. Violle, C. et al. (2012) The return of the variance: intraspecific variability in community ecology. Trends Ecol. Evol. 27, 244-252.

42. Dunne, J.A. et al. (2002) Network structure and biodiversity loss in food webs: robustness increases with connectance. Ecol. Lett. 5, 558-567.

43. Kaiser-Bunbury, C.N. et al. (2010) The robustness of pollination networks to the loss of species and interactions: a quantitative approach incorporating pollinator behaviour. Ecol. Lett. 13, 442-452.

44. Werner, E.E. and Gilliam, J.F. (1984) The ontogenetic niche and species interactions in size-structured populations. Annu. Rev. Ecol. Syst. 15, 393-425.

45. Temeles, E.J. et al. (2000) Evidence for ecological causation of sexual dimorphism in a hummingbird. Science 289, 441-443.

46. Radloff, F.G. and Du Toit, J.T. (2004) Large predators and their prey in a southern African savanna: a predator's size determines its prey size range. J. Anim. Ecol. 73, 410-423.

47. Del Hoyo, J. et al. (1994) Handbook of the birds of the world. Vol. 2. Lynx Editions.

48. Cramp, S. (1998) The complete birds of the Western Paleartic. CD-ROM. Oxford University Press.

49. Herrera, C.M. and Bazaga, P. (2013) Epigenetic correlates of plant phenotypic plasticity: DNA methylation differs between prickly and nonprickly leaves in heterophyllous Ilex aquifolium (Aquifoliaceae) trees. Bot. J. Linn. Soc. 171, 441452.

50. Lynch, M. and Walsh, B. (1998) Genetics and analysis of quantitative traits. Sinauer Associates, Inc. Publishers. 
51. Roby, G. et al. (2004) Berry size and vine water deficits as factors in winegrape composition: Anthocyanins and tannins. Australian Journal of Grape and Wine Research 10, 100-107.

52. González-Varo, J.P. et al. (2014) Who dispersed the seeds? The use of DNA barcoding in frugivory and seed dispersal studies. Methods Ecol. Evol. 5, 806-814.

53. Kuppler, J. et al. (2016) Time - invariant differences between plant individuals in interactions with arthropods correlate with intraspecific variation in plant phenology, morphology and floral scent. New Phytol., doi: 10.1111/nph.13858.

54. Herrera, C.M. (1984) A study of avian frugivores, bird-dispersed plants, and their interaction in mediterranean scrublands. Ecol. Monogr. 54, 2-23.

55. González-Castro, A. et al. (2012) Seed dispersal interactions in the Mediterranean Region: contrasting patterns between islands and mainland. J. Biogeogr. 39, 19381947.

56. Bartomeus, I. et al. (2016) A common framework for identifying linkage rules across different types of interactions. Funct. Ecol., doi: 10.1111/1365-2435.12666.

57. Polis, G.A. et al. (1989) The ecology and evolution of intraguild predation: Potential competitors that eat each other. Annu. Rev. Ecol. Syst. 20, 297-330.

58. Scharf, F.S. et al. (2000) Predator size-prey size relationships of marine fish predators: interspecific variation and effects of ontogeny and body size on trophicniche breadth. Mar. Ecol. Prog. Ser. 208, 229-248.

59. Woodward, G. and Hildrew, A.G. (2002) Body - size determinants of niche overlap and intraguild predation within a complex food web. J. Anim. Ecol. 71, 1063-1074.

60. Janssen, A. et al. (2002) Interspecific infanticide deters predators. Ecol. Lett. 5, 490-494.

61. Modha, M. (1967) The ecology of the Nile crocodile (Crocodylus niloticus laurenti) on Central Island, Lake Rudolf. Afr. J. Ecol. 5, 74-95.

62. Pienaar, U.d.V. (1969) Predator-prey relationships amongst the larger mammals of the Kruger National Park. Koedoe 12, 108-176.

63. Somaweera, R. et al. (2013) The role of predation in shaping crocodilian natural history. Herpetol. Monogr. 27, 23-51.

64. Griffin, A.S. (2016) Innovativeness as an emergent property: a new alignment of comparative and experimental research on animal innovation. Philos. Trans. $R$. Soc. Lond., Ser. B: Biol. Sci. 371.

65. Laland, K.N. and Reader, S.M. (1999) Foraging innovation in the guppy. Anim. Behav. 57, 331-340.

66. Tinker, M.T. et al. (2008) Food limitation leads to behavioral diversification and dietary specialization in sea otters. Proceedings of the National Academy of Sciences 105, 560-565. 
67. Lefebvre, L. et al. (2016) Feeding innovations in a nested phylogeny of Neotropical passerines. Philos. Trans. R. Soc. Lond., Ser. B: Biol. Sci. 371.

68. Aplin, L.M. et al. (2015) Experimentally induced innovations lead to persistent culture via conformity in wild birds. Nature 518, 538-541.

69. Rowntree, V.J. et al. (1998) Increased harassment of right whales (Eubalaena australis) by kelp gulls (Larus dominicanus) at Peninsula Valdes, Argentina. Mar. Mamm. Sci. 14, 99-115.

70. Marón, C.F. et al. (2015) Increased wounding of Southern Right Whale (Eubalaena australis) calves by Kelp Gulls (Larus dominicanus) at Penlnsula Valdés, Argentina. PLOS ONE 10, e0139291.

71. Schluter, D. and Grant, P.R. (1984) Ecological correlates of morphological evolution in a Darwin's finch, Geospiza difficilis. Evolution, 856-869.

72. Vander Wall, S.B. (2010) How plants manipulate the scatter-hoarding behaviour of seed-dispersing animals. Philos. Trans. R. Soc. Lond., Ser. B: Biol. Sci. 365, 989997.

73. Jansen, P.A. et al. (2012) Thieving rodents as substitute dispersers of megafaunal seeds. Proc. Natl. Acad. Sci. USA 109, 12610-12615.

74. Praz, C.J. et al. (2008) Host recognition in a pollen-specialist bee: evidence for a genetic basis. Apidologie 39, 547-557.

75. Kress, W.J. et al. (2015) DNA barcodes for ecology, evolution, and conservation. Trends Ecol. Evol. 30, 25-35.

76. Herrera, C.M. (1984) Adaptation to frugivory of Mediterranean avian seed dispersers. Ecology 65, 609-617.

77. Sobral, M. et al. (2010) Fruit-size preferences in wild and naive Eurasian blackbirds (Turdus merula) feeding on oneseed hawthorn (Crataegus Monogyna). The Auk 127, 532-539.

78. Costa, J.M. et al. (2016) Sampling completeness in seed dispersal networks: When enough is enough. Basic Appl. Ecol. 17, 155-164.

79. Migliore, J. et al. (2012) From Mediterranean shores to central Saharan mountains: key phylogeographical insights from the genus Myrtus. J. Biogeogr. 39, 942-956.

80. Jordano, P. (1987) Patterns of mutualistic interactions in pollination and seed dispersal: connectance, dependence asymmetries, and coevolution. The American Naturalist 129, 657-677. 
Figure I - Box 1
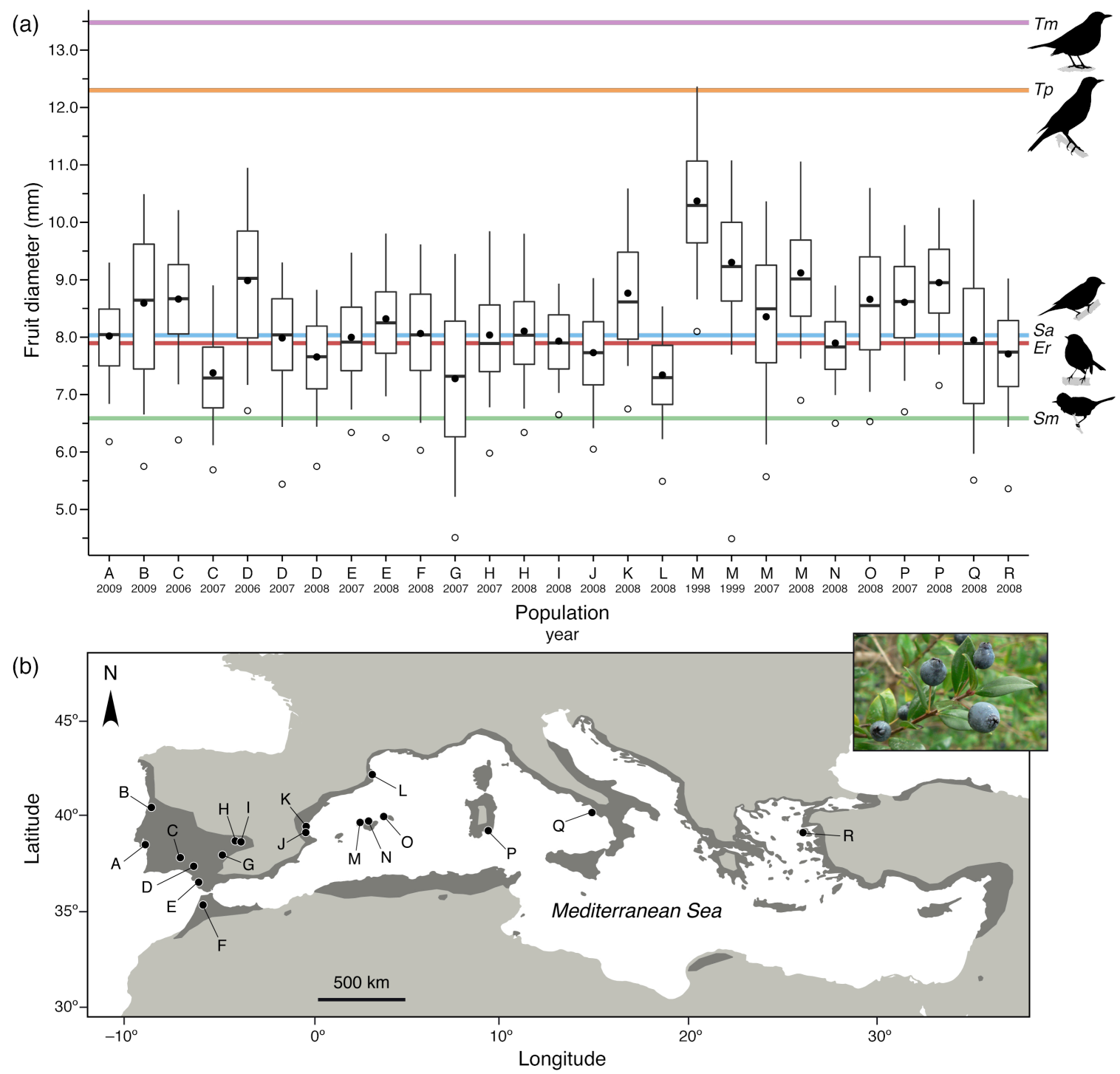
Figure II - Box 1

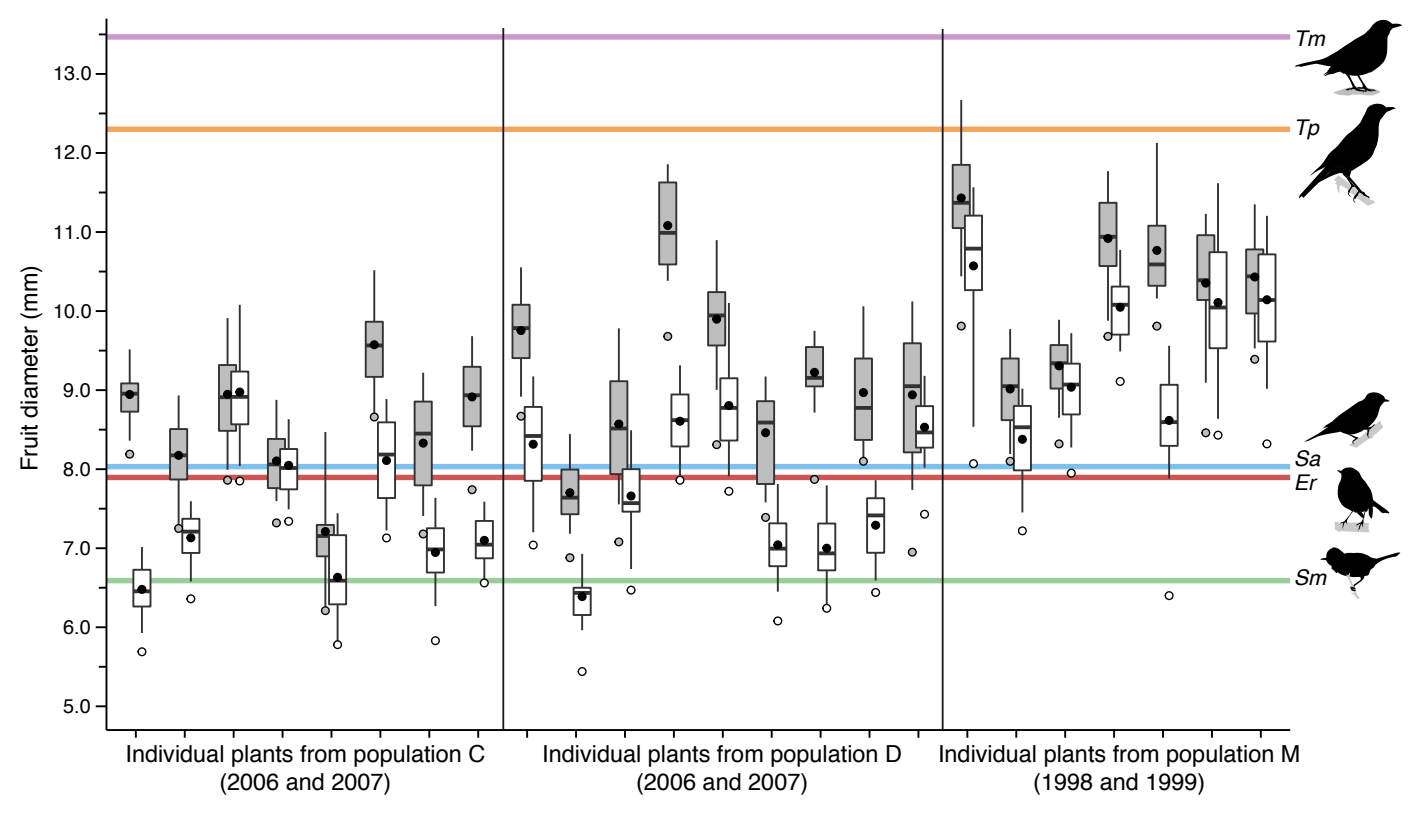


Figure 1

(a) totally forbidden link

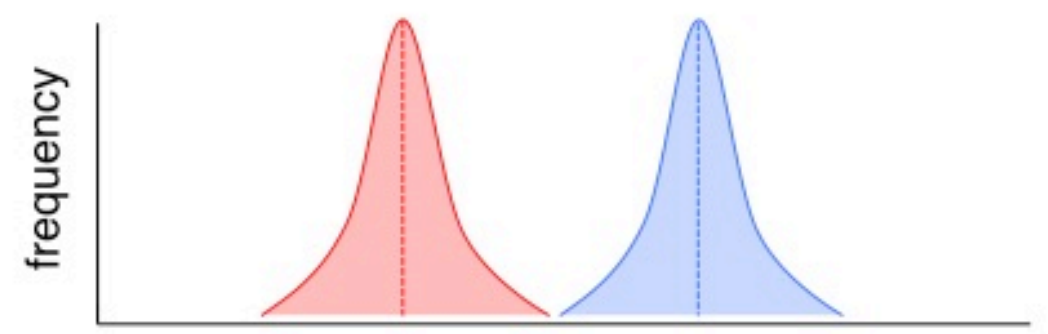

(b) partially forbidden link

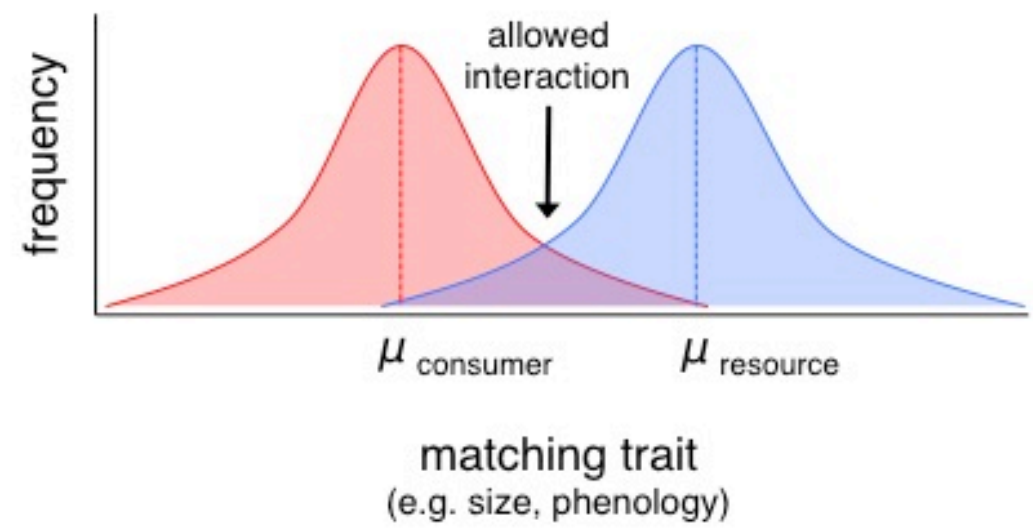


Figure 2
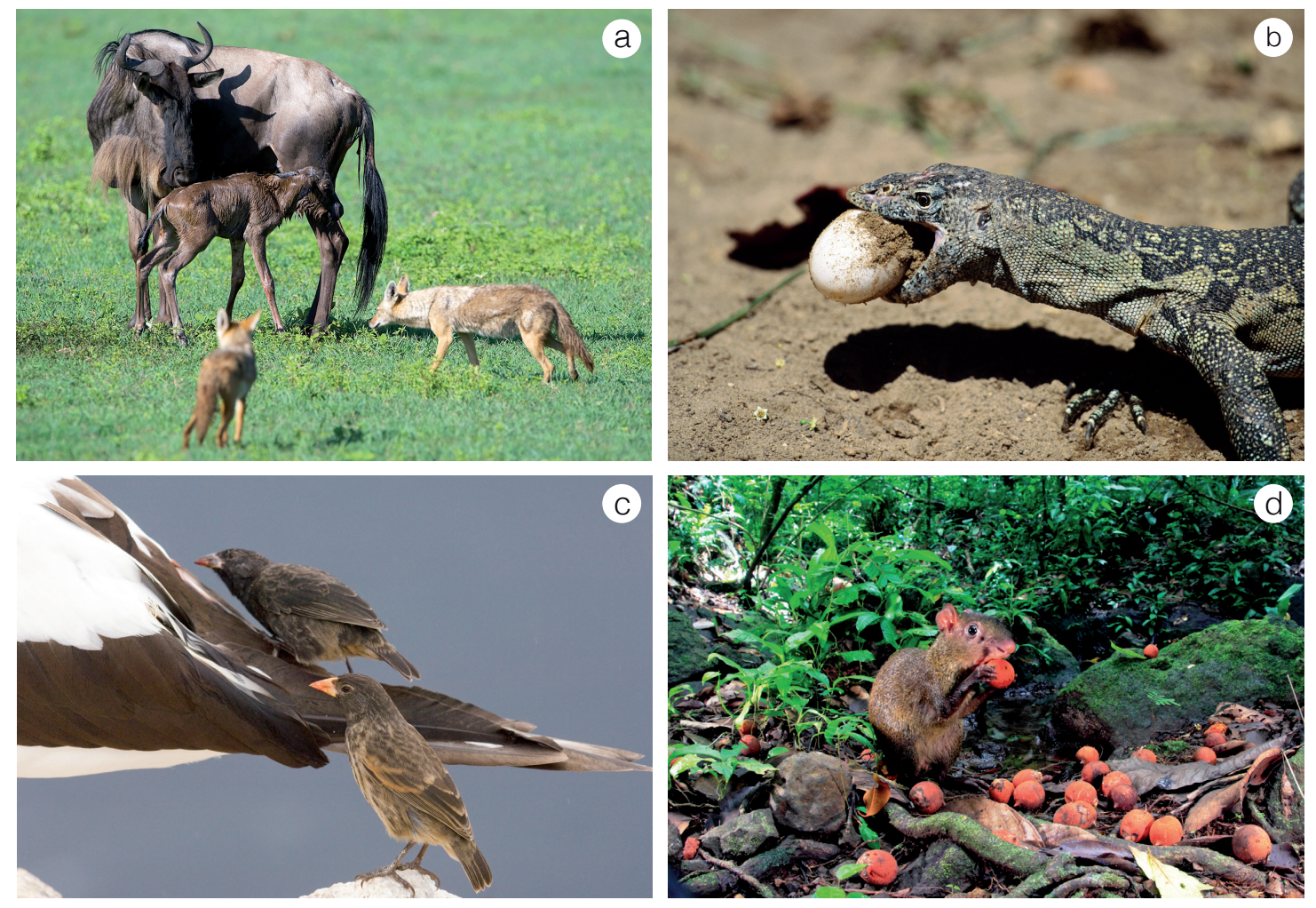
Figure 3

species $j \quad$ species $k$

(a)

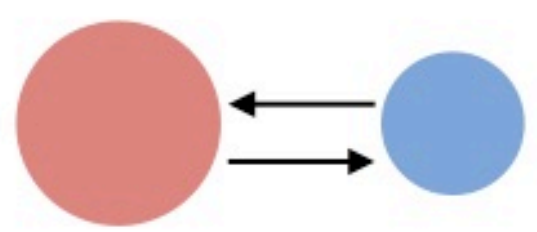

(b)

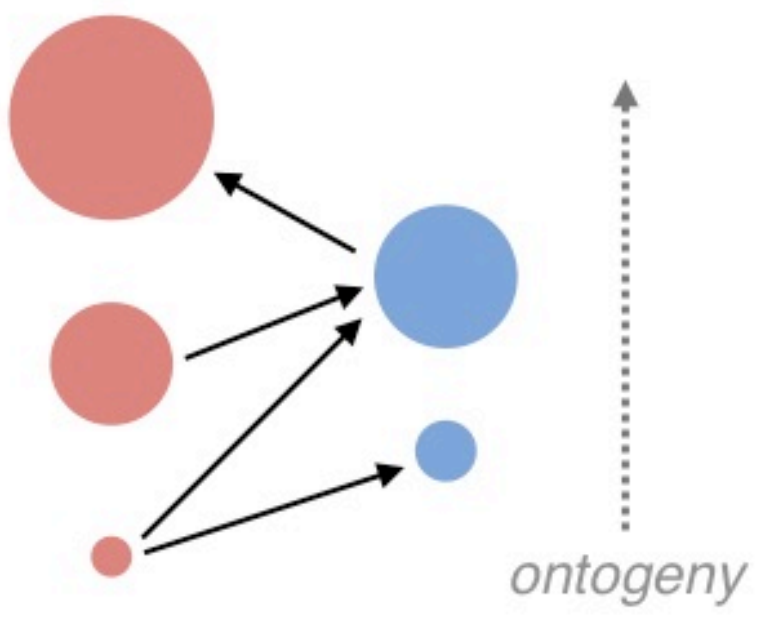

